\title{
AN INTERPOLATION THEOREM IN SYMMETRIC FUNCTION $F$-SPACES
}

\author{
H. HUDZIK AND L. MALIGRANDA
}

(Communicated by Palle E. T. Jorgensen)

\begin{abstract}
It is well known that every separable or perfect symmetric Banach function space $X$ is an interpolation space between $L^{1}$ and $L^{\infty}$ (see [1] and [4]). In this paper we prove that every symmetric function $F$-space is interpolation between $L^{0}$ and $L^{\infty}$, where $L^{0}$ is the space of all measurable functions whose support has finite measure. Moreover, for any function $f \in$ $L^{0}+L^{\infty}$ the norm $\|f\|_{L^{0}}+L^{\infty}$ is computed in the terms of the nonincreasing rearrangement function $f^{*}$ of $f$ as well as in terms of its distribution function $d_{f}$.
\end{abstract}

\section{INTRODUCTION}

In the sequel $(I, m)$ will denote the Lebesgue measure space on $I=[0,1]$ or on $I=[0, \infty)$. By $S=S(I, m)$ we mean the space of all equivalence classes of measurable real-valued functions defined on $I$.

Assume that $E=E(m)$ is a subgroup of $S(I, m)$ endowed with a functional \|\|$_{E}: E \rightarrow \mathbb{R}_{+}$that is even vanishing only at zero and satisfying the triangle inequality. Then $E$ is said to be a function $F^{*}$-group. A function $F^{*}$-group $\left(E,\|\|_{E}\right)$ such that $E$ is a linear space and the operation of multiplication by scalars is continuous is called a function $F^{*}$-space. Any function $F^{*}$-group ( $F^{*}$-space) that is a complete metric space with respect to the metric $d(f, g)=$ $\|f-g\|_{E}$ is called a function $F$-group ( $F$-space). For details concerning $F$ spaces see [7].

By $L^{0}=L^{0}(m)$ we shall denote the space of all measurable functions defined on $I$ whose support has finite measure. This space is endowed with the groupnorm

$$
\|f\|_{0}=m(\text { suppf }), \text { where suppf }=\{t \in I: f(t) \neq 0\} .
$$

By $L^{\infty}=L^{\infty}(m)$ we shall mean the Banach space of all $m$-essentially bounded functions endowed with the norm $\|f\|_{\infty}=\operatorname{ess}_{t \in I} \sup |f(t)|$.

Received by the editors March 20, 1989.

1980 Mathematics Subject Classification (1985 Revision). Primary 46E30, 46M35.

Key words and phrases. Symmetric function $F$-spaces, interpolation. 
The intersection and the algebraic sum of the spaces $L^{0}$ and $L^{\infty}$ shall be written as $L^{0} \cap L^{\infty}$ and $L^{0}+L^{\infty}$, respectively. The norms in these spaces will be defined as follows

$$
\begin{aligned}
& \|f\|_{L^{0} \cap L^{\infty}}=\max \left\{\|f\|_{0},\|f\|_{\infty}\right\}, \\
& \|f\|_{L^{0}+L^{\infty}}=\inf \left\{\|g\|_{0}+\|h\|_{\infty}: f=g+h, g \in L^{0}, h \in L^{\infty}\right\} .
\end{aligned}
$$

The spaces $L^{0}$ and $L^{0} \cap L^{\infty}$ are function $F$-groups, and $L^{0}+L^{\infty}$ is a function $F$-space (all are symmetric, see below).

In $f \in S(I, m)$, then the distribution function $d_{f}$ is defined by

$$
d_{f}(s)=m(\{t \in I:|f(t)|>s\}), \quad s \geq 0 .
$$

The nonincreasing rearrangement of $f$ is given by

$$
f^{*}(t)=\inf \left\{s>0: d_{f}(s) \leq t\right\}, \quad \inf \phi=\infty .
$$

Clearly, $d_{f}$ and $f^{*}$ are nonincreasing right-continuous functions on $[0, \infty)$. Notice that if $m I=\infty$, then these functions may also take value $\infty$.

A function $F$-group ( $F$-space) $\left(E,\|\|_{E}\right)$ is called symmetric (or rearrangement invariant) if:

1. $g \in S, f \in E$, and $|g| \leq|f|$, a.e., imply $g \in E$ and $\|g\|_{E} \leq\|f\|_{E}$,

2. For any $g \in S$ equimeasurable with $f \in E$, i.e., if $d_{g}=d_{f}$, then one has $g \in E$ and $\|g\|_{E}=\|f\|_{E}$.

Conditions 1 and 2 are equivalent to the following:

1: If $g \in S, f \in E, g^{*}(t) \leq f^{*}(t)$, and all $t \in I$, then $g \in E$ and $\|g\|_{E} \leq\|f\|_{E}$. Some properties of symmetric function $F$-spaces have been studied in [3].

\section{IMBEDDING THEOREM AND EXAMPLES}

Our first result is related to the problem given at the end of [2].

Theorem 1. (a) If $E$ is a nontrivial symmetric function $F$-group then

$$
L^{0} \cap L^{\infty} \subset E .
$$

(b) If $E$ also satisfies one of the following conditions:

(i) $\left\|1_{A}\right\|_{E}<\infty$ implies $m A<\infty$, or

(ii) $E$ is a symmetric function $F$-space,

then

$$
E \subset L^{0}+L^{\infty}
$$

Proof. (a) Let $0 \neq f \in E$. Define $A_{n}=\left\{t \in I:|f(t)| \geq \frac{1}{n}\right\}, n=1,2, \ldots$. We have $A_{n} \uparrow$ and $\cup_{n=1}^{\infty} A_{n}=$ suppf. Thus, $m A_{k}>0$ for some $k \in \mathbb{N}$. Since $k^{-1} 1_{A_{k}} \leq|f|$, we have $k^{-1} 1_{A_{k}} \in E$. By the assumption on $E$ we have $1_{A_{k}} \in E$. Now, let $g \in L^{0} \cap L^{\infty}$. There exist pairwise disjoint sets $B_{1}, B_{2}, \ldots, B_{\ell}$ such that $m\left(B_{i}\right)=m\left(A_{k}\right)$ for $i=1,2, \ldots, \ell$ and suppg $\subset \bigcup_{i=1}^{\ell} B_{i}$. All functions 
$1_{B_{i}}(i=1,2, \ldots, \ell)$ and $1_{A_{k}}$ are pairwise equimeasurable. Since, in view of our assumption, $E$ is symmetric, we have

$$
\left\|1_{B_{1}}\right\|_{E}=\left\|1_{B_{2}}\right\|_{E}=\cdots\left\|1_{B_{\ell}}\right\|_{E=}\left\|1_{A_{k}}\right\|_{E}
$$

Hence

$$
\| 1 \text { suppg }\left\|_{E} \leq\right\| \sum_{i=1}^{\ell} 1_{B_{i}}\left\|_{E} \leq \sum_{i=1}^{\ell}\right\| 1_{B_{i}}\left\|_{E}=\ell\right\| 1_{A_{k}} \|_{E}<\infty
$$

and so $\|g\|_{E} \leq\|\| g\left\|_{\infty} 1_{\text {suppg }}\right\|_{E} \leq\left(\left[\|g\|_{\infty}\right]+1\right)\left\|1_{\text {suppg }}\right\|_{E}<\infty$, where $\left[\|g\|_{\infty}\right]$ denotes the integer part of the number $\|g\|_{\infty}$. Thus, $g \in E$ and the inclusion (1) is proved.

(b) Assume first that $E$ satisfies condition (i). Let $f \in E$ and $A=\{t \in$ $I:|f(t)| \leq 1\}$. Then $f 1_{A} \in L^{\infty}$ and

$$
\left\|1_{I \backslash A}\right\|_{E} \leq\left\|f 1_{I \backslash A}\right\|_{E} \leq\|f\|_{E}<\infty .
$$

Thus, in view of (i), $m(I \backslash A)<\infty$, i.e., $f 1_{I \backslash A} \in L^{0}$.

Now assume that $E$ satisfies condition (ii) and $f \in E$. Define $A_{n}=\{t \in$ $I:|f(t)| \geq n\}, n=1,2, \ldots$. We shall prove that $m A_{k}<\infty$ for some $k \in \mathbb{N}$. In fact, if not, then we have $m A_{n}=\infty$ for any $n \in \mathbb{N}$. Choose $B_{n} \subset A_{n}$ with $m B_{n}=1$ for any natural $n$. All functions $1_{B_{n}}$ are equimeasurable, and so

$$
0 \leq\left\|1_{B_{1}}\right\|_{E}=\left\|1_{B_{n}}\right\|_{E} \leq\left\|\frac{1}{n} f 1_{B_{n}}\right\|_{E} \leq\left\|\frac{1}{n} f\right\|_{E} \rightarrow 0 \text { as } n \rightarrow \infty .
$$

Thus, $\left\|1_{B_{1}}\right\|_{E}=0$, a contradiction. Since $f 1_{A_{k}} \in L^{0}$ and $f 1_{I \backslash A_{k}} \in L^{\infty}$, the proof is finished.

Remarks. (1). O'Neil proved in [5] that the symmetric Orlicz group $L^{\psi}$ generated by generalized Young function $\psi$ satisfies $L^{0} \cap L^{\infty} \subset L^{\psi} \subset L^{0}+L^{\infty}$. Moreover, $L^{\psi}$ is an $F$-space, i.e., the scalar multiplication is continuous in $L^{\psi}$ if and only if $\lim _{u \rightarrow 0} \psi(u)=0$.

(2). Let us note that in (b) it can be obtained that if $f \in E$ then for every $\varepsilon>0$ there are functions $f_{1} \in L^{\infty}$ and $f_{2} \in L^{0}$, with $f_{1}+f_{2}=f$ such that $\left\|f_{1}\right\|_{\infty} \leq \varepsilon$ in the first case and $\left\|f_{2}\right\|_{0} \leq \varepsilon$ in the second one.

Example 1. Let $E=S$ on $I=[0, \infty)$ with the $F$-group norm

$$
\left.\|f\|_{E}=\frac{m(\text { suppf })}{1+m(\text { suppf })} \quad \text { (taking by definition } \frac{\infty}{1+\infty}=1\right) \text {. }
$$

Then $E$ does not satisfy any condition (i) and (ii) from Theorem 1, and $E \not \subset$ $L^{0}+L^{\infty}$.

Thus, it is interesting to pose the following problem:

Is the alternative of conditions (i) and (ii) necessary for (2)? Now, we give examples of symmetric function $F$-spaces and $F$-groups connected with Orlicz and Marcinkiewicz spaces. 
Example 2. Let $\psi$ be an Orlicz function, i.e., a continuous increasing function on $[0, \infty)$ such that $\psi(0)=0$. The Orlicz space $L^{\psi}$ is the space of all $f \in S$ such that $I_{\psi}(\lambda f)=\int_{I} \psi(\lambda|f(t)|) \mathrm{dm}<\infty$ for some $\lambda>0$ depending on $f$. The functional $\|\cdot\|_{\psi}$ defined on $L^{\psi}$ by $\|f\|_{\psi}=\inf \left\{\lambda>0: I_{\psi}(f / \lambda) \leq \lambda\right\}$ is an $F$-norm. Define a new Orlicz function $\psi$ by

$$
\psi(u)=\frac{\varphi(u)}{1+\varphi(u)} .
$$

Then $L^{\psi}=L^{0}+L^{\varphi}$ and $\|f\|_{\psi} \leq\|f\|_{+} \leq 4\|f\|_{\psi}$, where

$$
\|f\|_{+}=\inf \left\{\left\|f_{0}\right\|_{0}+\left\|f_{1}\right\|_{\varphi}: f=f_{0}+f_{1}, f_{0} \in L^{0}, f_{1} \in L^{\varphi}\right\} .
$$

In fact, from the inequality $\psi(u) \leq \min (1, \varphi(u))$ we have $L^{0} \subset L^{\psi}$ and $L^{\varphi} \subset L^{\psi}$, which imply $L^{0}+L^{\varphi} \subset L^{\psi}$ and the first inequality on the $F$-norms. On the other hand, if $f \in L^{\psi}$ and $\|f\|_{\psi}<\lambda$ then $I_{\psi}(f / \lambda) \leq \lambda$, and if $A=\left\{t \in I:|f(t)|>\lambda \varphi^{-1}(1)\right\}$, then

$$
\frac{m A}{2} \leq I_{\psi}\left(f 1_{A} / \lambda\right) \leq I_{\psi}(f / \lambda) \leq \lambda .
$$

Hence $f 1_{A} \in L^{0}$ and

$$
I_{\varphi}\left(f 1_{I \backslash A} / \lambda\right) \leq 2 I_{\psi}\left(f 1_{I \backslash A} / \lambda\right) \leq 2 I_{\psi}(f / \lambda) \leq 2 \lambda,
$$

i.e., $f 1_{I \backslash A} \in L^{\varphi}$. Thus $f \in L^{0}+L^{\varphi}$ and $\|f\|_{+} \leq\left\|f 1_{A}\right\|_{0}+\left\|f 1_{I \backslash A}\right\|_{\varphi} \leq$ $m A+2 \lambda \leq 4 \lambda$.

Example 3. Let $\psi$ be an Orlicz function. Define the Marcinkiewicz space $M^{\psi}$ generated by functional

$$
\|f\|_{M^{\psi}}=\inf \left\{\lambda>0: \sup _{t>0} \frac{f^{*}(t)}{\psi^{-1}(\lambda / t)} \leq \lambda\right\}
$$

Then $M^{\psi}$ is the symmetric function $F$-group. It is sufficient to prove the triangle inequality for $\|\cdot\|_{M^{\psi}}$. If $\|f\|_{M^{\psi}}<\lambda_{1}$ and $\|g\|_{M^{\psi}}<\lambda_{2}$, then $f^{*}(t) \leq$ $\lambda_{1} \psi^{-1}\left(\lambda_{1} / t\right)$ and $g^{*}(t) \leq \lambda_{2} \psi^{-1}\left(\lambda_{2} / t\right)$ for any $t>0$. Hence, by the property of rearrangement and the above,

$$
\begin{aligned}
(f+g)^{*}(t) & \leq f^{*}\left(\frac{\lambda_{1}}{\lambda_{1}+\lambda_{2}} t\right)+g^{*}\left(\frac{\lambda_{2}}{\lambda_{1}+\lambda_{2}} t\right) \\
& \leq \lambda_{1} \psi^{-1}\left(\frac{\lambda_{1}+\lambda_{2}}{t}\right)+\lambda_{2} \psi^{-1}\left(\frac{\lambda_{1}+\lambda_{2}}{t}\right)
\end{aligned}
$$

and so $\|f+g\|_{M^{\psi}} \leq \lambda_{1}+\lambda_{2}$, which gives the triangle inequality. Let us note that

and

$$
\|f\|_{M^{\psi}}=\inf \left\{\lambda>0: \sup _{s>0} \psi(s / \lambda) d_{f}(s) \leq \lambda\right\}
$$

$$
\|f\|_{M^{\psi}} \leq\|f\|_{\psi} \text { for } f \in L^{\psi}
$$


$M^{\psi}$ is a symmetric function $F$-space, i.e., the scalar multiplication is continuous in $M^{\psi}$ if and only if $\psi^{-1}$ satisfies the $\Delta_{2}$-condition (see [5, Theorem 9.9]). Moreover, $M^{\psi}(0,1)$ is separable if and only if $\lim _{u \rightarrow \infty} \psi(u)=c<\infty$ and $M^{\psi}(0, \infty)$ is not separable (see [5, Theorems 9.14 and 9.15]).

\section{AN INTERPOLATION THEOREM}

The first result is closely connected with the approximation spaces introduced by Peetre and Sparr in [6].

Proposition 1. Let $f \in S$ and $0 \leq s, t<m I$. Then

$$
f^{*}(t)=\inf \left\{\|f-g\|_{\infty}:\|g\|_{0} \leq t\right\}=\inf \left\{\left\|f-f 1_{A}\right\|_{\infty}: m A \leq t\right\}
$$

and

$$
d_{f}(s)=\inf \left\{\|f-h\|_{0}:\|h\|_{\infty} \leq s\right\} .
$$

Proof. Let $f^{*}(t)<\infty$ and $B=\left\{x \in I:|f(x)|>f^{*}(t)\right\}$. Then $m B=$ $d_{f}\left(f^{*}(t)\right) \leq t$ and

$$
\begin{aligned}
E(t, f): & =\inf \left\{\|f-g\|_{\infty}:\|g\|_{0} \leq t\right\} \\
& \leq \inf \left\{\left\|f-f 1_{A}\right\|_{\infty}: m A \leq t\right\} \\
& \leq\left\|f-f 1_{B}\right\|_{\infty}=\left\|f 1_{I \backslash B}\right\|_{\infty} \leq f^{*}(t) .
\end{aligned}
$$

If $f^{*}(t)=\infty$ then $m I=\infty$ and $f \notin L^{\infty}$. Hence for any $g \in L^{0}$ with $\|g\|_{0} \leq t$, we have $f-g \notin L^{\infty}$ and $E(t, f)=\infty$. Conversely, if $E(t, f)<\infty$, then for any $\varepsilon>0$ there exists $g \in L^{0}$ with $\|g\|_{0} \leq t$ such that $\|f-g\|_{\infty}<$ $E(t, f)+\varepsilon$. Assuming $\|f-g\|_{0}=u$ we get $|f|-|g| \leq|f-g| \leq u$, a.e. in $I$, and so

$$
\{x \in I:|f(x)|>u\} \subset\{x \in I:|g(x)|>0\} \cup A,
$$

where $A \subset I$ is a set of measure zero. Thus,

$$
d_{f}(u) \leq\|g\|_{0} \leq t \text { and } f^{*}(t) \leq u=\|f-g\|_{\infty}<E(t, f)+\varepsilon .
$$

Since $\varepsilon>0$ is arbitrary, the proof of (3) is complete. If $h \in L^{\infty}$ is such that $\|h\|_{\infty} \leq s$, then

$$
|f(x)-h(x)| \geq|f(x)|-|h(x)| \geq|f(x)|-s \text { a.e. in } I
$$

and so

$$
A_{s}=\{x \in I:|f(x)|>s\} \subset\{x \in I:|f(x)-h(x)|>0\} \cup A,
$$

where $A \subset I$ is a set of measure zero. Thus $d_{f}(s)=m A_{s} \leq\|f-h\|_{0}$. Moreover, taking $h_{0}=f 1_{I \backslash A_{s}}$ we have $\left\|h_{0}\right\|_{\infty} \leq s$ and $\left\|f-h_{0}\right\|_{0}=\left\|f 1_{A_{s}}\right\|_{0}=d_{f}(s)$. Thus

$$
\inf \left\{\|f-h\|_{0}:\|h\|_{\infty} \leq s\right\}=d_{f}(s) .
$$


Proposition 2. Let $E$ be a symmetric function $F$-space.

(a) If $A$ is a subset of $I$ of finite measure and $f, f_{n} \in E$ are such that $f_{n} \rightarrow f$ uniformly in $A \backslash B$, with $m B=0$, then $\left\|\left(f-f_{n}\right) 1_{A}\right\|_{E} \rightarrow 0$ as $n \rightarrow \infty$.

(b) The set of countable valued real functions is dense in $E$.

(c) An operator $\sigma_{a}(a>0)$ defined on $E$ by $\sigma_{a} f(t)=f(t / a)$ is continuous in $E$ and

$$
\left\|\sigma_{a} f\right\|_{E} \leq K_{a}\|f\|_{E}
$$

for any $f \in E$ and for a constant $K_{a}>0$ independent of $f$.

Proof. (a) From Theorem 1 and the fact that $m A$ is finite it follows that $1_{A} \in$ $E$. Now, the continuity of the multiplication operation yields

$$
\left\|\left(f-f_{n}\right) 1_{A}\right\|_{E} \leq\|\| f-f_{n}\left\|_{\infty} 1_{A}\right\|_{E} \rightarrow 0 \text { as } n \rightarrow \infty .
$$

(b) Let $f \in E$ and let $\left\{I_{n}\right\}_{n=1}^{\infty}$ be a sequence of pairwise disjoint sets of positive finite measure such that $\bigcup_{n=1}^{\infty} I_{n}=I$. For a given $\varepsilon>0$ and $n \in N$ there is a sequence $\left\{g_{m}^{n}\right\}_{m=1}^{\infty}$ of countable valued functions with supports in $I_{n}$ and such that $g_{m}^{n} \rightarrow f$ uniformly in $I_{n} \backslash A_{n}$ where $m A_{n}=0$, as $m \rightarrow \infty$. Thus, in view of (a), we have

$$
\left\|f 1_{I_{n}}-g_{m_{n}}^{n}\right\|_{E} \leq 2^{-n} \varepsilon
$$

for an $m_{n} \in \mathbb{N}, n=1,2, \ldots$. Denote, for brevity, $g_{m_{n}}^{n}$ by $g_{n}$ and define $g=\sum_{n=1}^{\infty} g_{n}$. Then

$$
\|f-g\|_{E}=\left\|\sum_{n=1}^{\infty}\left(f 1_{I_{n}}-g_{n}\right)\right\|_{E} \leq \sum_{n=1}^{\infty}\left\|f 1_{I_{n}}-g_{n}\right\|_{E} \leq \sum_{n=1}^{\infty} 2^{-n} \varepsilon=\varepsilon .
$$

(c) By virtue of (b), the proof proceeds in the same way as the proof of Theorem 4.4 in [4].

Theorem 2. Let $T: L^{0}+L^{\infty}$ be a homomorphism, i.e.,

$$
T(f+g)=T f+T g \text { and } T(-f)=-T f
$$

for any $f, g \in L^{0}+L^{\infty}$. Assume that $T$ maps $L^{0}$ into $L^{0}$ and $L^{\infty}$ into $L^{\infty}$, and

$$
\begin{aligned}
m(\text { suppTf }) & \leq M_{0} m \text { (suppf) } \quad \forall f \in L^{0}, \\
\|T f\|_{\infty} & \leq M_{1}\|f\|_{\infty} \quad \forall f \in L^{\infty} .
\end{aligned}
$$

Then $T$ maps any symmetric function $F$-space $E$ into itself and there exists a constant $M>0$ such that

$$
\|T f\|_{E} \leq M\|f\|_{E}
$$

for any $f \in E$.

Proof. First we shall prove that

$$
(T f)^{*}\left(M_{0} t\right) \leq M_{1} f^{*}(t)
$$

for any $f \in L^{0}+L^{\infty}$ and $t>0$. 
For this purpose note that for any $g \in L^{0}$ with $\|g\|_{0} \leq t$ we have $\|T g\|_{0} \leq$ $M_{0}\|g\|_{0} \leq M_{0} t$ and, in view of (3)

$$
(T f)^{*}\left(M_{0} t\right) \leq\|T f-T g\|_{\infty}=\|T(f-g)\|_{\infty} \leq M_{1}\|f-g\|_{\infty},
$$

i.e.,

$$
(T f)^{*}\left(M_{0} t\right) \leq M_{1} E(t, f)=M_{1} f^{*}(t) .
$$

Let $K>0$ be a constant such that $\left\|\sigma_{M_{0}} f\right\|_{E} \leq K\|f\|_{E}$ for any $f \in E$ (see Proposition 2(c)). Since each $f \in E$ belongs to $L^{0}+L^{\infty}$ (see Theorem 1) we can apply inequality (8) and we get $\|T f\|_{E}=\left\|(T f)^{*}\right\|_{E} \leq K\left\|\sigma_{1 / M_{0}}(T f)^{*}\right\|_{E} \leq$ $K\left\|M_{1} f^{*}\right\|_{E} \leq K\left(\left[M_{1}\right]+1\right)\left\|f^{*}\right\|_{E}=K\left(\left[M_{1}\right]+1\right)\|f\|_{E}$, where $\left[M_{1}\right]$ denotes the integer part of the number $M_{1}$. The proof is finished.

It is interesting, and useful as well, to describe the space $L^{0}+L^{\infty}$ and the $F$-norms $K(u, f)=\inf \left\{\|g\|_{0}+u\|h\|_{\infty}: f=g+h, g \in L^{0}, h \in L^{\infty}\right\},(u>0)$ of any function $f \in L^{0}+L^{\infty}$ in terms of its distribution function $d_{f}$ as well as in terms of its nonincreasing rearrangement $f^{*}$.

Proposition 3. The space $L^{0}+L^{\infty}$ consists of all functions $f$ in $S$ such that $d_{f}(s)<\infty$ for some $s>0$. Moreover, we have

$$
K(u, f)=\inf _{s>0}\left[s u+d_{f}(s)\right]=\inf _{t>0}\left[t+u f^{*}(t)\right] .
$$

Proof. Assume that $d_{f}(s)<\infty$ for scme $s>0$, i.e., $m A_{s}<\infty$ where $A_{s}=$ $\{x \in I:|f(x)|>s\}$. We have $f 1_{A_{s}} \in L^{0}, f 1_{I \backslash A_{s}} \in L^{\infty}, f=f 1_{A_{s}}+f 1_{I \backslash A_{s}}$ and so $f \in L^{0}+L^{\infty}$. Now, assume that $f \in L^{0}+L^{\infty}$, i.e., $f=g+h$ with $g \in L^{0}$ and $h \in L^{\infty}$. Let $s>2\|h\|_{\infty}$. Then

$$
\begin{aligned}
\{x \in I:|f(x)|>s\} & \subset\{x \in I:|g(x)|+h(x) \mid>s\} \\
& \subset\{x \in I:|g(s)|>s / 2\} \cup\{x \in I:|h(s)|>s / 2\} \\
& =\{x \in I:|g(x)|>s / 2\} \cup A .
\end{aligned}
$$

where $m A=0$. Hence, $d_{f}(s) \leq d_{g}(s / 2) \leq m$ (suppg) $<\infty$. If $f \in L^{0}+L^{\infty}$ then for any $\varepsilon>0$ there exists a decomposition $f=g+h$ such that $\|g\|_{0}+$ $u\|h\|_{\infty}<K(u, f)+\varepsilon$. Let $\|g\|_{0}=a$ and $\|h\|_{\infty}=b$. Then by Proposition 1,

$$
\begin{aligned}
\inf _{t>0}\left[t+u f^{*}(t)\right] & \leq a+u f^{*}(a) \\
& =a+u E(a, f) \leq a+u \widetilde{E}(a, f) \leq a+u\|h\|_{\infty} \\
& =\|g\|_{0}+u\|h\|_{\infty}<K(u, f)+\varepsilon,
\end{aligned}
$$

where $\widetilde{E}(a, f)=\inf \left\{\|f-g\|_{\infty}:\|g\|_{0}=a\right\}$, and

$$
\inf _{s>0}\left[s u+d_{f}(s)\right] \leq b u+d_{f}(b) \leq b u+\|g\|_{0}=\|g\|_{0}+u\|h\|_{\infty}<K(u, f)+\varepsilon .
$$

Since $\varepsilon>0$ was arbitrary, we have that $\inf _{t>0}\left[t+u f^{*}(t)\right]$ and $\inf _{s>0}\left[s u+d_{f}(s)\right]$ do not exceed $K(u, f)$. On the other hand, if $d_{f}\left(s_{0}\right)<\infty$, then for $s>s_{0}$ the 
measure of the sets $A_{s}=\{x \in I:|f(x)|>s\}$ is finite and their intersection has measure zero. Therefore $\lim _{s \rightarrow \infty} d_{f}(s)=0$ and so $f^{*}(t)<\infty$ for any $t>0$. Setting $A=\left\{x \in I:|f(x)|>f^{*}(t)\right\}$ we have $m A=d_{f}\left(f^{*}(t)\right) \leq t$ and

$$
K(u, f) \leq\left\|f 1_{A}\right\|_{0}+u\left\|f 1_{I \backslash A}\right\|_{\infty} \leq m A+u f^{*}(t) \leq t+u f^{*}(t)
$$

for any $t>0$. Thus

$$
K(u, f) \leq \inf _{t>0}\left[t+u f^{*}(t)\right]
$$

For any $\varepsilon>0$ there exists an $s_{0}>0$ such that $s_{0} u+d_{f}\left(s_{0}\right)<\inf _{s>0}\left[s u+d_{f}(s)\right]$ $+\varepsilon$. Then

$$
K(u, f) \leq\left\|f 1_{A_{s_{0}}}\right\|_{0}+u\left\|f 1_{I \backslash A_{s_{0}}}\right\|_{\infty} \leq d_{f}\left(s_{0}\right)+u s_{0}<\inf _{s>0}\left[s u+d_{f}(s)\right]+\varepsilon
$$

and (9) is proved.

Remarks. (3) Putting together the results in [6, Proposition 4.4] and Proposition 1 , we also get the proof of (9).

(4) All our results are also true for arbitrary measure space $(\Omega, \Sigma, \mu)$.

(5) Inequality $E(t, f) \leq \widetilde{E}(t, f)$ always holds and if the measure is nonatomic, then it is possible to prove even the equality $E(t, f)=\widetilde{E}(t, f)$.

\section{REFERENCES}

1. J. Bergh and J. Löfström, Interpolation spaces, an introduction, Springer-Verlag, Berlin, Heidelberg, and New York, 1976.

2. H. Hudzik, On the smallest and largest Orlicz spaces, Math, Nachr. 141 (1989), 109-115.

3. B. Kotkowski, Symmetric spaces I, II, Bull. Acad. Polon. Sci. Sér. Sci. Math. Astronom. Phys. 16 (1968), 871-875, 951-954.

4. S. G. Krein, Yu. I. Petunin, and E. M. Semenov, Interpolation of linear operators, Nauka, Moscow, 1978; English transl., Amer. Math. Soc., Providence, RI, 1982.

5. R. O'Neil, Integral transforms and tensor products on Orlicz spaces and $L(p, q)$-spaces, $\mathrm{J}$. Analyse Math. 21 (1968), 1-276.

6. J. Peetre and G. Sparr, Interpolation of normed Abelian groups, Ann. Mat. Pura Appl. 92 (1972), 217-262.

7. S. Rolewicz, Metric linear spaces, 2nd ed., PWN, Warsaw, 1984.

(H. Hudzik) Institute of Mathematics, University of A. Mickiewicz, Matejki 48/49, 60-769 PoznÁn, Poland

(H. Hudzik and L. Maligranda) Institute of Mathematics, Polish Academy of Sciences, MielżyńsKiego 27/29, 61-725 Poznán, Poland

(L. Maligranda) Departamento de Matemáticas, Instituto Venezolano de Investigaciones Científicas, Apartado 21827, Caracas 1020-A, Venezuela

(L. Maligranda) Departamento de Matemáticas, Facultad de Ciencias, Universidad Central de Venezuela, Apartado 20513, Caracas 1040-A, Venezuela 